\title{
Fabrication of alignment layer free flexible liquid crystal cells using thermal nanoimprint lithography
}

\author{
Tzu-Chieh Lin ${ }^{\mathrm{a}, *}$, Shao-Chi Yu ${ }^{\mathrm{a}}$, Pei-Shiang Chen ${ }^{\mathrm{a}}$, Kai-Yuan Chi ${ }^{\mathrm{a}}$, Han-Chang Pan ${ }^{\mathrm{b}}$, Chih-Yu Chao ${ }^{\mathrm{a}}$ \\ a Department of Physics, National Taiwan University, No. 1, Sec. 4, Roosevelt Road, Taipei 10617, Taiwan, ROC \\ ${ }^{\mathrm{b}}$ Instrument Technology Research Center, National Applied Research Laboratories, 20 RED Road VI, Hsinchu 30076, Taiwan, ROC
}

\section{A R T I C L E I N F O}

\section{Article history:}

Received 16 April 2008

Received in revised form 18 May 2008

Accepted 20 May 2008

Available online 6 June 2008

\section{PACS:}

61.30.-V

61.30.Gd

Keywords:

Thermal nanoimprint lithography

Flexible LCD

\begin{abstract}
A B S T R A C T
In this article, thermal nanoimprint lithography (thermal-NIL) has been utilized to transfer the microgroove pattern onto the plastic substrate. Without coating alignment polymer, the microgrooves on the flexible substrates can align liquid crystals (LCs) directly. The flexible LC cell is shown to maintain comparable electro-optical properties while bending. The plastic film and the alignment layer integrated into an alignment substrate could effectively prevent cracks of the additional polymer alignment layer during the bending process. This method is applicable to the roll-to-roll process to increase the production efficiency.
\end{abstract}

(c) 2008 Elsevier B.V. All rights reserved.

\section{Introduction}

Many flexible displays based on plastic substrates have been designed and developed in recent years. They are taken to be the mainstream displays for next generation displays. The applications range from hand-held electronic products such as smart cards, electronic books, watches to wearable displays, image-rendering wall hangings, etc. Compared with traditional liquid crystal displays (LCDs) using glass substrates, these novel alternatives have attracted large attention due to several merits such as flexibility, light weight, thin profile, high shock resistance, and high portability. Among them, four leading prototypes are E-ink [1], electrophoretic display (EPD) [2], organic light emitting diode display (OLED) [3], and LCDs [4-9]. E-ink has low power consumption and high contrast ratio, but it is not suitable for color displays. Comparing to the EPD, the LCD has faster response time and lower driving voltage to be a motion image display. Furthermore, the material of LCD has better stability and lower cost than that of OLED. Thus, the applications of LCDs using plastic substrates are burgeoning now-a-days. Even so, aligning LCs uniformly on plastic substrates is still a crucial issue investigated by many groups in the world [4-9]. In many flexible LCDs, for producing microgrooves, polymer films were still utilized to be coated on plastic substrates to be the alignment layers [4-7]. But the substrates coated with polymer alignment layers

\footnotetext{
* Corresponding author.

E-mail address: tclin@phys.ntu.edu.tw (T.-C. Lin).
}

are bended for many times in practice, the polymer layer will easily get ruptured during the bending process. In addition, a high-temperature baking process (about $250^{\circ} \mathrm{C}$ ) of forming a rigid alignment film is a serious problem causing thermal distortion for plastic substrates. Furthermore, a number of studies have been proposed that maintaining LC alignment can also be achieved via the formation of polymer networks [8,9]. However, residual polymers of unexposed region result in lower transmittance and higher operating voltage. Therefore, to make the flexible materials serve not only as substrates but also as alignment layers will be a very critical topic for the fabrication of flexible LCDs.

In this paper, we fabricate grating microgrooves directly on the surface of flexible substrates to align LCs immediately by using thermal-NIL. Without coating the polymer alignment layers, the alignment layer free substrates can avoid cracks of the traditional LC alignment layer during the bending process. The flexible LC device fabricated by this method is found to possess lower driving voltage and comparable response time compared to the traditional LCDs. Furthermore, our method suggests that one could fabricate multifunctional substrates for flexible LCDs without additional polymer alignment layers.

\section{Experimental details}

The flexible substrate we choose in this study is polyethylene terephthalate (PET) film, which is a kind of transparent plastic with some good properties like high transmittance, low coefficient of 


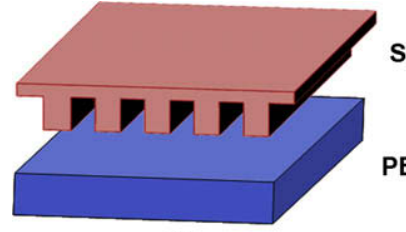

a

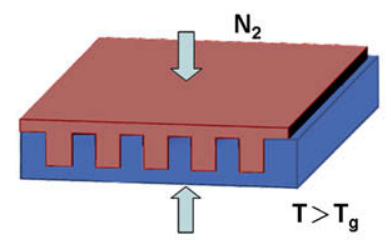

b

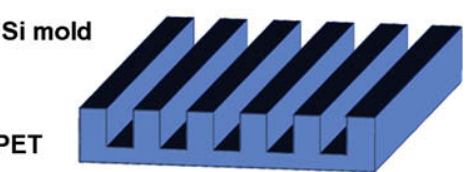

C

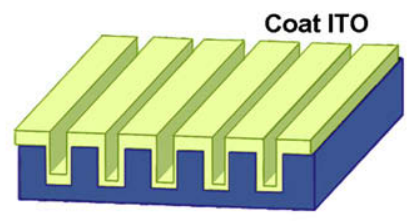

d
Fig. 1. Thermal nanoimprint lithography (thermal-NIL) method. (a)Heating the chamber results in a molten surface on the PET substrate, (b) press the mold and PET substrate together by high-pressure nitrogen gas, (c) remove the mold and the bare PET with microgrooves was obtained and (d) coat ITO thin film as an electrode on the PET substrate.

thermal expansion, stable mechanical property, good chemical resistance, and so on. According to these advantages, PET films are used for touch panels widely. The surface of the PET substrate is required to be flat before NIL. After being cleaned mildly in ultrasonic with ethanol and deionized water, the PET film was dried out by nitrogen gas. Then the plastic films were prepared completely for the next step, thermal-NIL. Fig. 1 shows the flowchart for the thermal-NIL process. The mold for NIL in this study is the silicon wafer with microgrooves patterned by the traditional microlithography process. The PET film was placed on the silicon mold in the chamber and then they were pressed together at the pressure of about 260 psi when the chamber was heated slightly above the $T_{\mathrm{g}}\left(\sim 78^{\circ} \mathrm{C}\right)$ of PET. This thermal-NIL enables the pattern of the mold to be replicated onto the plastic film via controlling the sys- tem under the proper temperature and pressure. The microgroove nanostructures of the PET film after nanoimprinting were measured by atomic force microscope (AFM), as shown in Fig. 2a. Fig. $2 \mathrm{~b}$ shows the scanning electron microscope (SEM) image of the surface structure of the PET. The width of the groove is about $500 \mathrm{~nm}$. To avoid the high working temperature over $T_{\mathrm{g}}$ of PET, we use RF magnetron sputter[10] to coat a thin indium tin oxide (ITO) layer as an electrode on the microgroove surface of the PET at room temperature. After coating such thin ITO layer, the depth of grooves is about $60 \mathrm{~nm}$ (see Fig. 2c), which is still good enough for LC molecules alignment. Two patterned PET substrates were arranged to be a flexible $90^{\circ} \mathrm{TN}$ cell. The cell gap of the LC cell is $1.5 \mu \mathrm{m}$ and the LC material is a nematic LC. After sealing the substrates by UV glue, the PET flexible substrates could still adhere to each other well against bending [11].

\section{Electro-optical measurement and discussions}

By setting the polarizer and the analyzer to be crossed, the $90^{\circ}$ TN cell is normally white as shown in Fig. 3a. Fig. 3b shows the cell driven at $3 \mathrm{~V}$, the LCs are aligned vertically with applied electric field and thus make no phase retardation for the incident light. Under the crossed polarizers, the LC cell is at the dark state. In Fig. 4, the electro-optical (EO) switching curve was measured under flat and three different bending conditions. These TN LC cells are set to be normally black under parallel polarizers. The transmissions of dark state under different bending conditions are almost the same. As the bending curvature increases, the bright state becomes worse. This could be resulted from the reduction of cell gap and certain disorder of LC molecules alignment when the LC cell is bent [12]. This problem could be overcome by forming the polymer wall. However, even the curve radius is small as $2.3 \mathrm{~cm}$, the transmittance still remains $70 \%$ of the flat one. Besides, the threshold voltage does not alter under flat and different bending conditions. The threshold voltages of these flexible LC cells without polymer alignment layers are around $1.2 \mathrm{~V}$, even lower than that of the tra- a

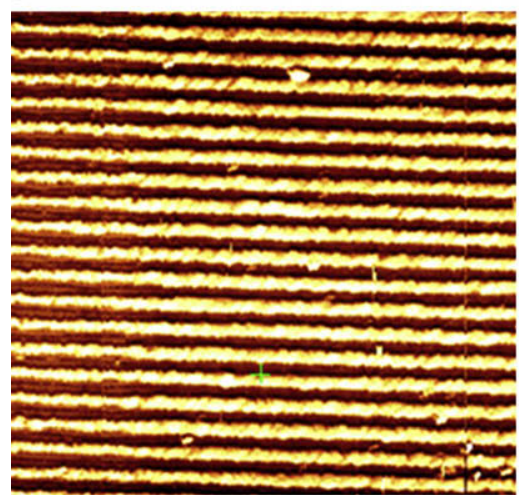

b

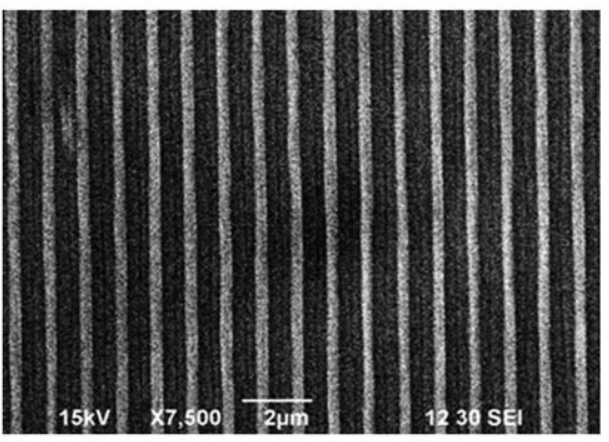

C

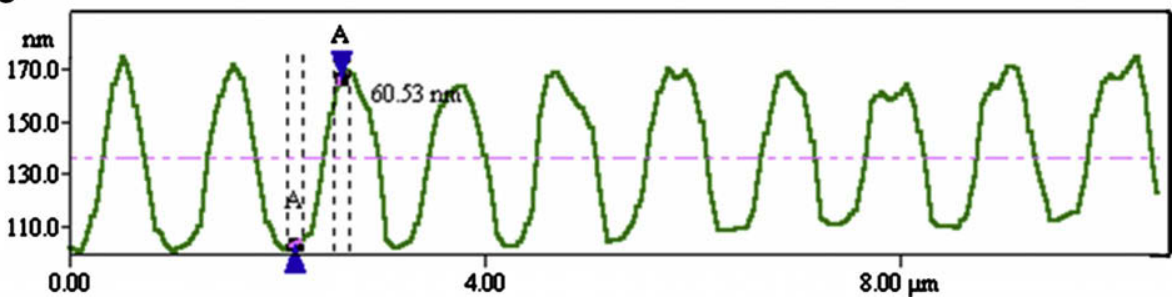

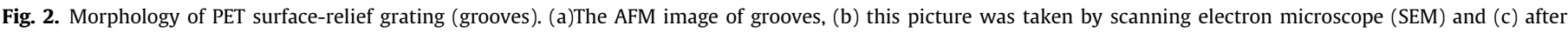
coating an ITO film, the height of grooves measured by AFM was approximately $60 \mathrm{~nm}$. 
a

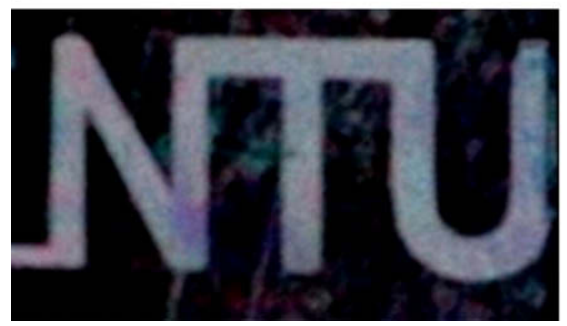

b

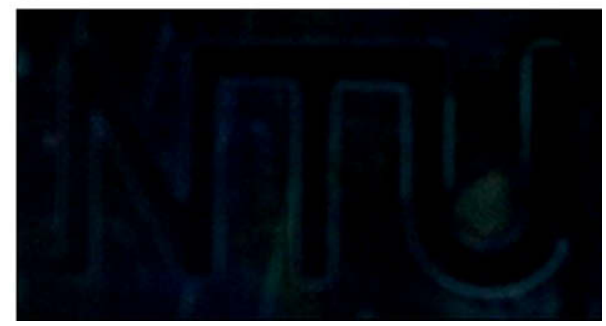

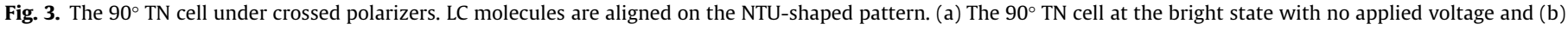
with applied voltage about $3 \mathrm{~V}$, the TN cell is at dark state due to LCs align vertically with applied electric field.

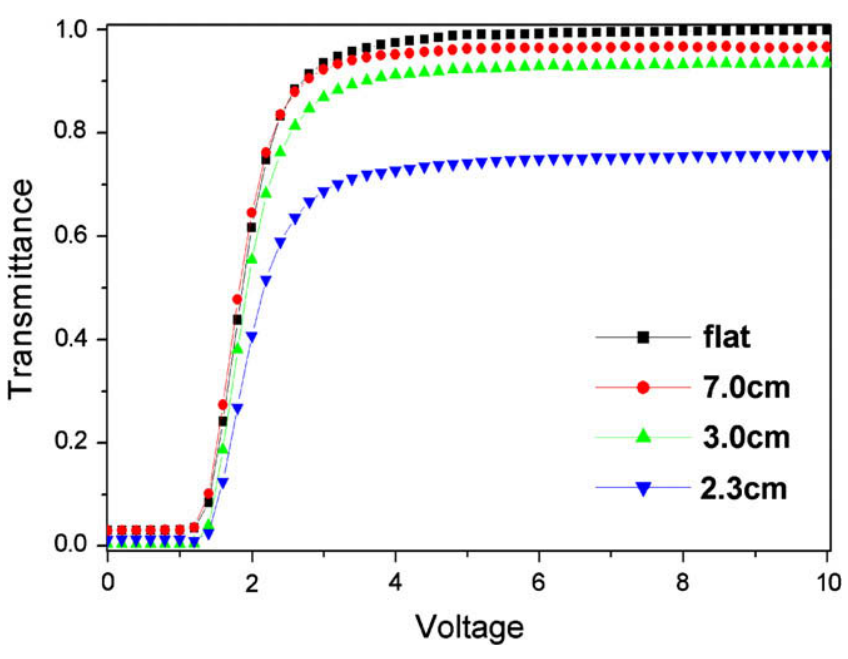

Fig. 4. Electro-optical switching curves of our flexible TN LC cell under four different bending conditions. These TN LC cells are normally black with parallel polarizers. As the curvature increases, the transmittance of bright state gets lower. But even the curve radius is small as $2.3 \mathrm{~cm}$, the transmittance still remains $70 \%$ of the flat one.

Table 1

When the applying voltage is 10 volt, the response time is measured around $13 \sim 15$ $\mathrm{ms}$ at these curvatures, which is fast enough for a motion image display. The optical performances are comparable to that of the traditional LC cells with glass substrates

\begin{tabular}{lll}
\hline Bending radius $(\mathrm{cm})$ & Threshold voltage $(\mathrm{V})$ & Response time $(\mathrm{ms})$ \\
\hline Flat & 1.2 & 13.1 \\
$7 \mathrm{~cm}$ & 1.2 & 15.3 \\
$3 \mathrm{~cm}$ & 1.2 & 15.1 \\
$2.3 \mathrm{~cm}$ & 1.2 & 13.2 \\
\hline
\end{tabular}

ditional LCDs. This effect may be caused by the absence of the polymer alignment layer and the larger pitch of NIL-grooves than that of conventional rubbing [13]. When the applying voltage is $10 \mathrm{~V}$, the response time is measured around $13 \sim 15 \mathrm{~ms}$, which is fast enough for a motion image display (see Table 1 ). The optical performances of these flexible TN LC cells are comparable to that of the traditional LC cells using glass substrates.

\section{Conclusions}

By means of thermal-NIL technique, the microgroove pattern can be directly transferred on the plastic substrate, and thus LC molecules can be aligned immediately on the patterned flexible substrates without the need to have additional polymer coating layers for LC alignment. This method not only can avoid the cracks of the traditional alignment layer during bending process, but also increase the flexibility of rollable LC cells. The flexible TN LC cell of this design has the lower threshold voltage and comparable response time compared to the traditional LCDs. Moreover, when this flexible LC cell is bent, its threshold voltage and response time still remain the same. Utilizing the roll-to-roll process could make the fabrication of such flexible displays higher throughput and lower cost than the traditional rigid substrate LCDs. Finally, when the surprising conductive plastic material becomes more mature and more obtainable, the substrate, alignment layer, and the electrode could be combined into one. These non-laminated devices will raise the stability and the flexibility of the flexible LCD to be more practical rollable displays.

\section{Acknowledgements}

This work was supported by the National Science Council and Ministry of Education of the Republic of China. The authors would like to express their acknowledgements to Mr. Ta-Cheng Chien of Henkel Taiwan Corp. for his assistance in the development of this research.

\section{References}

[1] B. Comiskey, J.D. Albert, H. Yoshizawa, J. Jacobson, Nature 394 (1998) 253.

[2] A. Henzen, N. Ailenei, F.V. Reeth, G. Vansichem, R.W. Zehner, K. Amundson, SID Int. Symp. Digest Tech. Pap. 35 (2004) 1070.

[3] Y. Li, L.W. Tan, X.T. Hao, K.S. Ong, F. Zhu, L.S. Hung, Appl. Phys. Lett. 86 (2005) 153508.

[4] F. Matsumoto, T. Nagata, T. Miyabori, H. Tanaka, S. Tsushima, SID Int. Symp. Digest Tech. Pap. 28 (1993) 965.

[5] K.H. Liu, W.Y. Chou, C.C. Liao, C.T. Ho, H.P. Shieh, Jpn. J. Appl. Phys. 45 (2006) 7761.

[6] Y.T. Kim, J.H. Hong, T.Y. Yoon, S.D. Lee, Appl. Phys. Lett. 88 (2006) 263501.

[7] Y.T. Kim, S. Hwang, J.H. Hong, S.D. Lee, Appl. Phys. Lett. 89 (2006) 173506.

[8] H. Sato, H. Fujikake, Y. Iino, M. Kawakita, H. Kikuchi, Jpn. J. Appl. Phys. 41 (2002) 5302.

[9] J.P.A. Vogel, S.I. Klink, R. Penterman, H. de Koning, E.E.A. Huitema, D.J. Broer SID Int. Symp. Digest Tech. Pap. 35 (2004) 767.

[10] H.C. Pan, M.H. Shiao, C.Y. Su, C.N. Hsiao, J. Vac. Sci. Technol. A 23 (2005) 1187.

[11] S.C. Yu, Master Thesis National Taiwan University, (2007).

[12] Q. Wang, R. Guo, M.R. Daj, S.W. Kang, S. Kumar, Jpn. J. Appl. Phys. 46 (2007) 299.

[13] R. Lin, J.A. Rogers, Nano. Lett. 7 (2007) 1613. 